\title{
Short communication: Influence of pasteurization on the active compounds in medicinal plants to be used in dairy products
}

\author{
A. K. Jäger, ${ }^{1}$ L. Saaby, D. S. Kudsk, K. C. Witt, and P. Mølgaard \\ Department of Medicinal Chemistry, Faculty of Pharmaceutical Sciences, University of Copenhagen, 2 Universitetsparken, 2100 Copenhagen, \\ Denmark
}

\begin{abstract}
Interest from the dairy industry in adding herbal drugs to milk and yogurt products raises the question of whether these plant materials can be pasteurized. Root material of Rhodiola rosea, Eleutherococcus senticosus, and Panax ginseng, all plants with adaptogenic activities, was pasteurized. The content of active compounds in the root material before and after pasteurization was quantified by HPLC analysis. The results show that the eleutherosides in E. senticosus, and to an extent the ginsenosides from $P$. ginseng, could withstand pasteurization, whereas salidroside and rosavin from $R$. rosea did not survive pasteurization. Thus, $R$. rosea is not suitable for products requiring pasteurization.
\end{abstract}

Key words: Eleutherococcus senticosus, Panax ginseng, pasteurization, Rhodiola rosea

Producers in the dairy industry would like to introduce milk and yogurt products with beneficial health properties. Of interest are performance- and immuneboosting products that could contain plant material from adaptogenic plant species. Species such as Russian root (Eleutherococcus senticosus), ginseng root (Panax ginseng), and Arctic root (Rhodiola rosea) are well-known adaptogenic plants (Darbinyan et al., 2000; Davydov and Krikorian, 2000; ESCOP, 2003; Shevtsov et al., 2003; Persson et al., 2004; Panossian and Wagner, 2005; Reay et al., 2006). Panax ginseng contains a series of ginsenosides and E. senticosus several eleutherosides to which the adaptogenic activity is attributed. Both species are included in the European Pharmacopoeia (2008), where the monographs include quantitative assessment of the 2 groups of compounds. Rhodiola rosea contains salidroside, tyrosol, and rosavins, which are presumed to be the active compounds.

Received November 13, 2009.

Accepted March 15, 2010.

${ }^{1}$ Corresponding author: ankj@farma.ku.dk
Before plant material can be added to a milk-based product, it needs to be pasteurized. For practical reasons, it is most convenient to pasteurize the plant material separately before addition to the milk-based product, as is done with fruit. The heating process could influence the composition of the active substances in the immune-boosting plants. Previous studies on the effects of pasteurization have dealt mainly with the visual quality of the plant material, such as the color stability of fruit and vegetable additions (Vasquez et al., 2007; Gössinger et al., 2009a,b), but also with the antioxidant activity in tomatoes (Dede et al., 2007). One study investigated the effect of pasteurization on the antioxidant effect of the medicinal plant Echinacea purpurea (Chen et al., 2009). In the present study, we investigated the effect of pasteurization of the plant material on the concentration of the active compounds.

Root materials of Eleutherococcus senticosus Maxim (Araliaceae) and Panax ginseng C.A. Meyer (Araliaceae) were purchased from Natur Drogeriet (Hørning, Denmark). Roots of Rhodiola rosea L. (Crassulaceae) were collected from a cultivated field in Falster, Denmark, and dried at $40^{\circ} \mathrm{C}$. All root materials were ground to a powder.

For pasteurization, powdered root material was suspended in water $(1: 10 \mathrm{wt} / \mathrm{vol})$ at $95^{\circ} \mathrm{C}$ and kept at this temperature for $4 \mathrm{~min}$. The mixture was cooled to room temperature in an ice bath. The plant material was separated from the water phase by suction filtration. The plant material was dried at $37^{\circ} \mathrm{C}$ for $24 \mathrm{~h}$, and the water phase was lyophilized.

Pasteurized and nonpasteurized root material of E. senticosus was processed according to the assay in the European Pharmacopeia (2008) monograph for Eleutherococci radix. The lyophilized water phase from the pasteurization of $E$. sentocosus was redissolved in $50 \%$ ethanol and filtered through a $0.45-\mu \mathrm{m}$ nylon filter. Pasteurized and nonpasteurized root material of $P$. ginseng was processed according to the assay in the European Pharmacopeia monograph for Panax radix (European Pharmacopeia, 2008). The lyophilized water phase from the pasteurization of $P$. ginseng was redis- 
Table 1. Content of eleutherosides (\%) before and after pasteurization of Eleutherococcus senticosus root

\begin{tabular}{lccc}
\hline Sample & Eleutheroside B & Eleutheroside E & Total eleutherosides \\
\hline Nonpasteurized plant material & 0.035 & 0.010 & 0.045 \\
Pasteurized plant material & 0.002 & 0.017 & 0.019 \\
Water phase from pasteurization & 0.034 & 0.010 & 0.044 \\
\hline
\end{tabular}

Table 2. Content of ginsenosides (\%) before and after pasteurization of Panax ginseng root

\begin{tabular}{lccc}
\hline Sample & Ginsenoside Rg 1 & Ginsenoside Rb 1 & Total ginsenosides \\
\hline Nonpasteurized material & 0.222 & 0.473 & 0.695 \\
Pasteurized material & 0.042 & 0.275 & 0.317 \\
Water phase from pasteurization & 0.084 & 0.092 & 0.176 \\
\hline
\end{tabular}

solved in $20 \%$ acetonitrile and filtered through a $0.45-$ $\mu \mathrm{m}$ nylon filter.

One hundred milligrams each of pasteurized and nonpasteurized material of $R$. rhodiola was extracted with $3 \mathrm{~mL}$ of extraction solution (methanol:dichloromethane $1: 3$, containing $0.4 \mathrm{mg} / \mathrm{mL}$ salicin as internal standard) for $60 \mathrm{~min}$ in an ultrasound bath. The extract was centrifuged and $2 \mathrm{~mL}$ of supernatant transferred to a new vial. Two milliliters of the extraction solution was added to the plant material and the extraction process repeated. The plant material was extracted 3 times in total, resulting in $6 \mathrm{~mL}$ of supernatant, which was taken to dryness under vacuum. The extract was redissolved in $1 \mathrm{~mL}$ of $50 \%$ methanol and filtered through a $0.45-\mu \mathrm{m}$ nylon filter. The lyophilized water phase from the pasteurization of $R$. rhodiola was redissolved in $50 \%$ methanol and filtered through a $0.45-\mu \mathrm{m}$ nylon filter.

All pasteurization and extraction procedures were performed twice. Quantitative analysis of the eleutheroside content in the extracts from E. senticosus was carried out according to the procedure in the European Pharmacopoeia (2008) utilizing a Waters HPLC apparatus (Waters, Milford, MA) with a Waters 1525 pump system and a photodiode array detector (Waters

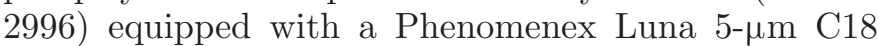
$250 \times 4.6 \mathrm{~mm}$ column (Phenomenex, Torrance, CA). Quantitative analysis of the ginsenoside content in the extracts from $P$. ginseng was carried out according to the procedure in the European Pharmacopoeia (2008) utilizing a Shimadzu HPLC apparatus (Kyoto, Japan) with a Shimadzu FCV 10AL pump system connected to a Shimadzu SPD-10 AV detector and equipped with Nucleosil 5- $\mu \mathrm{m}$ C18 $125 \times 4.6 \mathrm{~mm}$ column (MachereyNagel GmbH and Co. KG, Düren, Germany). Quantitative analysis of salidroside and rosavin was carried out on a Shimadzu SPD-6AV HPLC apparatus equipped with a Phenomenex Luna $5-\mu \mathrm{m} \mathrm{C18} 150 \times 4.6 \mathrm{~mm}$ column. The oven temperature was $40^{\circ} \mathrm{C}$. Elution was carried out with gradient elution with $\mathrm{A}$ (acetonitrile $5 \%$ ) and B (methanol 50\%) as follows: time 0 to $10 \mathrm{~min}$ with eluent B 0 to $66 \%$, time 10 to 12 min eluent B 66 to $100 \%$, time 12 to 15 min $100 \%$ eluent B. The flow rate was $1 \mathrm{~mL} / \mathrm{min}$; detection was carried out at 221 $\mathrm{nm}$. All determinations were done in duplicate.

Pasteurization of E. senticosus root material did not lead to a destruction of the eleutherosides, but the compounds were found in the water phase (Table 1). This means that either the entire pasteurization mixture should be used or adding the extract rather than the plant material should be considered. After pasteurization of $P$. ginseng root, ginsenosides were found in both the plant material and the water phase (Table 2). The combined ginsenosides in the pasteurization mixture constituted $72 \%$ of the ginsenosides found in nonpasteurized root material. The pasteurization of $R$. rosea root let to a loss of more than $80 \%$ of salidroside and $90 \%$ of rosavin (Table 3); what remained of the two compounds was found in the water phase.

The results show that Russian root, E. senticosus, can be pasteurized without loss of the active compounds, and ginseng root, $P$. ginseng, can withstand pasteurization with loss of about $25 \%$ of the active compounds. Both of these roots are suitable for preparations that involve pasteurization. On the other hand, the presumed active compounds in Arctic root, $R$. rosea, could not withstand the pasteurization procedure; thus, $R$. rosea not a candidate for addition to dairy products. These results highlight that plant materials with beneficial effects cannot simply be added to dairy products because the pasteurization process may destroy the effects of the plants.

Table 3. Contents (\%) of salidroside and rosavin before and after pasteurization of Rhodiola rosea root

\begin{tabular}{lcc}
\hline Sample & Salidroside & Rosavin \\
\hline Nonpasteurized material & 0.396 & 1.663 \\
Pasteurized material & 0.006 & 0.008 \\
Water phase from pasteurization & 0.078 & 0.131 \\
\hline
\end{tabular}




\section{REFERENCES}

Chen, X. M., C. Hu, and D. D. Kitts. 2009. Effect of high-pressure pasteurisation on the antioxidant activity of Echinacea purpurea root and flower. Pharm. Biol. 47:29.

Darbinyan, V., A. Kteyan, A. Panossian, E. Gabrielian, G. Wikman, and H. Wagner. 2000. Rhodiola rosea in stress induced fatigue a double blind cross-over study of a standardized extract SHR-5 with a repeated low-dose regimen on the mental performance of healthy physicians during night duty. Phytomedicine 7:365-371.

Davydov, M., and A. D. Krikorian. 2000. Eleutherococcus senticus (Rupr. \& Maxim) Maxim (Araliaceae) as an adaptogen: A closer look. J. Ethnopharmacol. 72:345-393.

Dede, S., H. Alpas, and A. Bayindirli. 2007. High hydrostatic pressure treatment and storage of carrot and tomato juices: Antioxidant activity and mircobial safety. J. Sci. Food Agric. 87:773-782.

ESCOP (European Scientific Cooperative on Phytotherapy). 2003 ESCOP Monographs - The Scientific Foundation for Herbal Medicinal Products. 2nd ed. Thieme Verlag, Stuttgart, Germany.

European Pharmacopoeia. 2008. Edition 6.2. European Directorate for the Quality of Medicines (EDQM), Council of Europe, Strasbourg, France.

Gössinger, M., S. Moritz, M. Hermes, S. Wendelin, H. Scherbichler, H. Halbwirth, K. Stich, and E. Berghofer. 2009b. Effects of processing parameters on colour stability of strawberry nectar from puree. J. Food Eng. 90:171-178.
Gössinger, M., T. Ullram, M. Hermes, S. Wendelin, S. Berghold, H. Halbwirth, K. Stich, and E. Berghofer. 2009a. Effects of prefreezing, puree content and pasteurisation regimen on colour stability of strawberry nectar made from puree. J. Sci. Food Agric. 89:144-149.

Panossian, A., and H. Wagner. 2005. Stimulating effect of adaptogens: An overview with particular reference to their efficacy following single dose administration. Phytother. Res. 19:819-838.

Persson, J., E. Bringlöv, L. G. Nilsson, and L. Nyberg. 2004. The memory-enhancing effects of Ginseng and Ginko biloba in healthy volunteers. Psychopharmacology 172:430-434.

Reay, J. L., D. O. Kennedy, and A. B. Scholey. 2006. Effects of Panax ginseng, consumed with and without glucose, on blood glucose levels and cognitive performance during sustained "mentally demanding" tasks. J. Psychopharmacol. 20:771-781.

Shevtsov, V. A., B. I. Zholus, V. I. Shervarly, V. B. Volskij, Y. P. Korovin, M. P. Khristich, N. A. Roslyakova, and G. Wikman. 2003. A randomized trial of two different doses of a SHR-4 Rhodiola rosea extract versus placebo and control of capacity for mental work. Phytomedicine 10:95-105.

Vasquez, A. L., S. Schilling, R. Carle, and S. Neidhart. 2007. Effects of thermal processing and fruit matrix on $\beta$-carotene stability and enzyme inactivation during transformation of mangoes into purée and nectar. Food Chem. 102:1172-1186. 